12 Weiner, L. P., et al., New England fournal of Medicine, 1972, 286, 385. 13 Sweet, B. H., and Hilleman, M. R., Proceedings of the Society for Experimental Biology and Medicine, 1960, 105, 420.

14 Shah, K. V., Ozer, H. L., Pond, H. S., Palma, L. D., and Murphy, G. P., Nature, 1971, 231, 448.

16 Spencer, E. S., and Andersen, H. K., British Medical fournal, 1970, 3, 251.

\section{Urinary Tract Infection in Newborn}

Infection of the urinary tract in infancy and childhood is a dangerous condition. In about half the cases radiological investigation shows an abnormality such as pyelonephritic scarring or vesicoureteric reflux..$^{2}$ It may be associated with progressive renal damage unless the infection is controlled.

The peak incidence is during the first 4 weeks of life, ${ }^{3}$ and at this age mortality is high during the acute stage of the illness. But despite the possibility of reliable diagnosis from examination of the urine and effective treatment with antibiotics cases probably still remain undetected. The problem is that at this age the symptoms are varied and most of them are nonspecific.

In a recent review ${ }^{4}$ of 66 infants with urinary tract infection in the first month of life J. M. Littlewood found that the commonest features were low weight, pyrexia, lethargy, and gastrointestinal symptoms such as anorexia, vomiting, and loose stools. Though the kidneys were palpable in more than half the patients, in only some were they thought to be enlarged. In no case did a macroscopic abormality of the urine or an observed disorder of micturition draw attention to the diagnosis. The more serious symptoms such as dehydration, irritability, and convulsions were found in only a minority of patients, probably as a result of early diagnosis in this series. Jaundice, which occurred in $11 \%$ of the cases, has been reported before as a result of renal tract infection, ${ }^{5}$ but could be a trap for the unwary. Boys were more often affected than girls, and predisposing factors were an abnormal mode of delivery, perinatal anoxia, and low or high birth weight.

It is not easy to obtain uncontaminated urine from infants, and quantitive culture ${ }^{6}$ is especially useful in this age group. However, obvious bacteriuria on simple microscopy of fresh uncentrifuged urine correlates well with a count of more than 100,000 organisms per $\mathrm{ml}$ on culture. The problem of contamination can be overcome by obtaining urine by suprapubic puncture, and provided conditions are suitable this is a safe techniques in infants. ${ }^{7}$ Littlewood also confirmed the well-known facts that neither the absence of proteinuria nor the absence of pyuria excludes the diagnosis of infection.

The severity of the illness in newborn babies in contrast to adult patients was emphasized by the finding of a positive blood culture in $30 \%$ and a blood urea higher than $50 \mathrm{mg} / 100 \mathrm{ml}$ in over half of the patients. The overall mortality was $11 \%$. Two of the deaths occurred in infants with major congenital abnormalities of the urinary tract incompatible with survival, while the other five were of patients treated some years ago by means (alkalis and sulphonamides) which would be considered inadequate now. No deaths occurred in the latter part of the series, when ampicillin was the drug of choice and streptomycin or chloramphenicol were used for the more severely ill infants. Pyelonephritis in infancy can cause acute renal failure, which may be completely reversible and peritoneal dialysis has been used successfully in this situation. ${ }^{8}$
The clinical features of neonatal urinary infection may be classed in four groups: firstly, a severe systemic illness, often with septicaemia, and with clinical evidence of pyelonephritis; secondly, infection secondary to a major congenital abnormality of the urinary tract; thirdly, mild nonspecific symptoms with no signs to incriminate the renal tract; and, fourthly, asymptomatic infants in whom infection of the urine is an incidental finding. The clinical spectrum is so wide that urinary tract infection should be suspected in any newborn baby whose progress is in any way abnormal, and examination of the urine should be carried out as a routine.

1 Smellie, J. M., Hodson, C. J. Edwards, D., and Normand, I. C. S., British Medical fournal, 1964, 2, 1222.

Rolleston, G. L., Shannon, F.' T., and Utley, W. L. F., British Medical fournal, $1970,1,460$.

Stansfeld, J. M., British Medical fournal, 1966, 1, 631

4 Littlewood, J. M., Archives of Disease in Childhood, 1972, 47, 218. Arthur, A. B., and Wilson, B. D. R., British Medical fournal, 1967, $1,539$.

6 Kass, E. H., Transactions of the Association of American Physicians,

7 1956, 69, 56.

8 Meadow, S. R., Cameron, J. S., Ogg, C. S., and Saxton, H. M., Archives of Disease in Childhood, 1971, 46, 221.

\section{International Cancer Research}

The permanent building of the International Agency for Research on Cancer (I.A.R.C.), a gift of the French, was formally opened in Lyons during June 1972. To mark the event the Agency's annual report for $1971^{1}$ includes comments on general developments since it moved to its temporary quarters five years ago. From the outset the study of the role of environmental factors in the aetiology of human cancers has been central to the Agency's activities. Studies in cancer diagnosis and control remained the province of the cancer unit of the World Health Organization, with which a close liaison was established early on.

Organizing research on an international basis is expensive. Language barriers and politics are apt to add to the intrinsic problems of the research itself. But the research in the I.A.R.C.'s programme is essentially international. It was sensible for the Agency, therefore, to concentrate on cancer epidemiology, because the time was ripe for studies of cancer incidence in remote communities and on migrants from one country to another. The appropriate methods based on the use of computers had been developed, and there remain parts of the world still relatively unaffected by such features of modern Western life as motor cars, air pollution, and prepacked and processed foods.

The Agency has five units based in Lyons: epidemiology and biostatistics, environmental carcinogenesis, biological carcinogenesis, chemical carcinogenesis, and research training. In addition it helps to support regional centres in Nairobi, Singapore, and Jamaica. The fact that information reaches Lyons from cancer registries all over the world has enabled the I.A.R.C. to suggest improvements in the international classification of diseases. At the same time the Agency has been able to draw attention to cancer problems peculiar to particular communities or geographical areas-for example, the high incidence of laryngeal and hypopharyngeal cancers in Thailand among smokers of "keeyo," a mixture of tobacco and other substances. The publication ${ }^{2}$ of the proceedings of a symposium on cancer and other chronic diseases in 
migrants to Israel was of particular interest. The Agency has been especially active in the fields of asbestos carcinogenesis, liver cancer, ${ }^{3}$ and cancer of the oesophagus.

Events have partly overtaken the Agency's efforts to determine whether DDT is a carcinogen for man or not. Exposure of mice to diets containing DDT increases the risk of their developing liver tumours, but the results are diffcult to interpret because mice of many strains, especially males, are peculiarly prone to develop liver tumours. Moreover, DDT appears to decrease the risk of the development of other tumours such as lymphomas. An experimental study on rats is not sufficiently advanced for any conclusion to be drawn. Meanwhile the use of DDT has been banned in the United States. In many parts of the world where the Agency and W.H.O. operate DDT is an important weapon against malaria and various insect pests. Banning its use in such areas would seriously increase the risk of non-neoplastic disease and decrease food production. At present the cancer hazard for man from DDT is purely theoretical. On the basis of a preliminary evaluation of its own field studies the Agency has concluded that there seems to be "no correlation between DDT exposure and primary liver cancer or cancer at other sites." It will be interesting to see what stand the Agency and W.H.O. take in relation to DDT.

1 International Agency for Research on Cancer, Annual Report, 1971, p. 128. Lyons, World Health Organization, 1972.

Davies, A. M., and Sacks, M., Israel fournal of Medical Sciences, 1971, 7, 1331 .

International Agency for Research on Cancer, Liver Cancer. Lyons, I.A.R.C. Scientific Publications No. 1, 1971.

\section{Thymectomy for Myasthenia Gravis}

The discovery of an association between the thymus and myasthenia gravis was originally based on two findings. Firstly, A. Blalock and colleagues ${ }^{1}$ found in 1936 that thymectomy in a myasthenic patient led to a considerable improvement in her symptoms. Secondly, patients with the disease were found to have in common a histological change in the thymus. This was the presence of germinal centres and plasma cells in the gland, suggesting an immune reponse in the thymus, which does not normally show one.

Germinal centres and antibody production in the thymus can occur in animals if the antigen is directly injected into it. Moreover, the offspring of myasthenic mothers have transient myasthenia from birth, which disappears within a few weeks. Apparently there is a transmissible agent able to cross the placenta and cause the disease in the infant. The effect lasts long enough to make it unlikely that it is a small molecule, and it is reasonable to suppose, but unproved, that it is an IgG antibody. This would cross the placenta, and the duration of the effect in the child is commensurate with its half life. It thus seems that myasthenia may be the result of an immune response in the thymus leading to the production of antibody which can interfere with neuromuscular transmission.

It is difficult to see how an antibody can interfere directly with the action of a neurotransmitter, as the space across which the transmitter acts is smaller than the diameter of an IgG molecule. Biopsies of myasthenic muscle have shown IgG bound to the muscle in a striated pattern, not associated with the neuromuscular junction. While immunization of animals with thymic tissue results in an antibody with affinity for muscle, the antibody also reacts well with smooth muscle and seems unlikely to have the selective effect of extra fatiguability of voluntary muscle seen in myasthenia. Just as much of the antibody produced may be made at the site of injection of antigen, so the germinal centres in the thymus may be evidence of a local response to local thymic antigen. The nature of the antigen, whether endogenous or an infecting agent located in the thymus, remains completely unknown.

The effect of thymectomy in producing improvement in the disease suggests that the process does not normally exist to any extent outside the thymus. It is now well established that the thymus-dependent lymphocytes which are required for most humoral antibody responses need the thymus in which to mature. Thymectomy in the adult leads to depletion of these cells after about 200 days. The improvement in the disease which frequently occurs sooner than this is unlikely to be due to their depletion; it is more likely that it is due to the removal of the source of production of the antibody itself.

It used to be believed that improvement after thymectomy was seen most frequently in young girls. ${ }^{2}$ Subsequently this was shown to be due to the infrequency of enlargement of the thymus in this group. Thus these girls had total thymectomy, whereas other patients had enucleation of an obviously abnormal part of the gland from a remainder that was also abnormal. A recent report ${ }^{3}$ on a large series of treated thymectomy patients shows that the improvement rate is very high when total thymectomy is carried out, though many of the patients did not show improvement for many months after operation. The authors related the rate of response to the number of germinal centres in the gland: the more frequent the centres, the slower the remission. Early or late remissions were stable ones.

It appears that the remissions after surgery in myasthenia may be of two kinds-rapid response by removal of the antibody-producing tissue, and a more delayed response when depletion of $\mathrm{T}$ cells in the body prevents the abnormal response in other tissues. Thus thymectomy, which is clearly worthwhile in severe myasthenia, may be both eradicating a localized process in the thymus and in some patients also stopping a more remote production of antibody.

\footnotetext{
1 Blalock, A., Mason, M. F., Morgan, H. J., and Riven, S. S., Annals of Surgery, 1939, 110, 544.

2 Eaton. L. M., and Clagett, O. T., American fournal of Medicine, 1955, 19, 703.

3 Papatestas, A. E., Alpert, L. I., Osserman, K. E., Osserman, R. S., and Kark, A. E., American 'fournal of Medicine, 1971, 50, 465.
}

\section{Surgical Ritual}

In the complex and rapidly evolving organization of modern surgical wards and operating theatres it is inevitable that many procedures have to be made "routine." Indeed it is highly desirable that patients have routine screening of chest and blood before surgery, that consent forms are signed, that identification labels are firmly fixed, and that a hundred other tasks are performed. Yet a routine must never become a senseless ritual which persists because it has become hallowed by tradition-the tourniquet has at last disappeared from the foot of the bed of every amputation case-and should exist only if it is based on sound reasons which can be easily understood by both medical and nursing staff. As knowledge and techniques advance, 